\begin{abstract}
ASTIN COLLOQUIUM I966 ARNHEM SUBJECT TWO

APPLICATIONS OF METHODS OF OPERATIONS RESEARCH AND MODERN ECONOMIC THEORY
\end{abstract}




\title{
APPLICATIONS OF METHODS OF OPERATIONS RESEARCH AND MODERN ECONOMIC THEORY
}

\author{
Introductory report \\ J. VAN KLINKEN \\ Amsterdam
}

It is rather a pity that we received only three papers especially devoted to subject 2, "applications of methods of operations research and modern economic theory". Maybe the time for real applications of operations research techniques in the insurance field has, still to come. However, I am convinced that sooner or later such techniques-in connection with the use of the computor-will play a more or less important part in the management of insurance business. An impulse to this of course will be the appearance of -let me say-"textbooks" on this subject; such as the book written by Wolff, Methoden der Unternehmensforschung im Versicherungswesen, Springerverlag I966, a copy of which I received a few days ago.

Meanwhile, the fact that only a few papers have been sent in gives me the opportunity to report on them in some detail and to make a few side remarks.

The studies presented are:

Borch. Dynamic decision problems in an insurance company. de Leve and Weeda. Driving with Markov-programming.

d' Hooge, Franckx and Gennart. Utilisation pratique de la méthode de simulation dans l'assurance "non-life".

\section{Prof. Karl Borch. Dynamic decision problems in an insurance company}

The paper Prof. Borch submitted, deals essentially with some problems related with random walk theory. Partly it can be considered to be the counterpart of the classical discontinuous ruin theory as its subject is the expected duration of life of the company and maximisation of the discounted value of dividend payments. 
The question of calculating the expected duration of life and more specially the means the company has to influence its future lifetime involves reinsurance and utility theory to which topic in recent years Prof. Borch has devoted a number of very interesting publications.

The rather alternative problem treated by Borch, the determination of the optimal dividend policy, belongs typically to the domain of "operations research".

The contribution of Borch to this ASTIN-colloquium is in my opinion a very valuable one. The problems dealt with are interesting and the mathematical treatment is lucid and instructive. I consider it a pleasure and an honour to be in the opportunity to report on this paper.

Permit me now to review the article in some detail. Borch starts with a rather simple random walk model for the capital $S$ of the company at the end of the subsequent years. The classical ruin theory considered only the possibility of $S$ becoming zero or negative; this means the introduction of an absorbing barrier $S=0$. This model implies that the company never will pay any dividends and is therefore not so realistic. As Borch remarks this has been pointed out already by de Finetti in I957. It is sensible to add to the model a reflecting barrier $S=Z$ and to assume that the excess $s_{t}=S_{t}-Z$ in year $t$ is paid out at once as dividend. Furthermore the supposition is made that the profits of subsequent years are independently distributed with the same distribution function $F(x)$.

\section{Expected life of the company}

Let $S$ denote the initial capital of the company, $Z$ the reflecting barrier and $D(S, Z)$ the expected number of years before ruin occurs. For $o \leq S \leq Z$ the function $D(S, Z)$ must satisfy the integral equation of Fredholm's type:

$D(S, Z)=\mathrm{I}+\{\mathrm{I}-F(Z-S)\} D(Z, Z)+\int_{0}^{z} D(x, Z) f(x-S) d x$,

with $f(x)$ the derivative of the profit distribution function $F(x)$. This integral equation has a unique solution given by the LiouvilleNeumann expansion. Hence we may assume that for a given profit distribution $F(x)$, the function $D(S, Z)$ is known. Now 
suppose that the only aim the company strives for is to survive as long as possible. To this end the company may accept and compare reinsurance contracts. If the company accepts an amount $P$ for a reinsurance contract with claim distribution $G(y)$, this deal is a favourable one in the case:

$$
\int_{0}^{\infty} D(S+P-y) d G(y)>D(S, Z) .
$$

Moreover the company will prefer contract $A$ to contract $B$ if:

$$
\int_{0}^{\infty} D\left(S+P_{A}-y\right) d G_{A}(y)>\int_{0}^{\infty} D\left(S+P_{B}-y\right) d G_{B}(y) .
$$

By means of the function $D(S, Z)$ we obtain a preference ordering for reinsurance contracts. $D(S, Z)$ can be interpreted as a utility function, which, owing to the special problem dealt with, has the feature of being completely defined. We here have virtually an application of utility theory.

Maximum of the expected discounted value of dividend-payments

The second problem Borch considers is the determination of the value of the reflecting barrier $Z$ which makes the expected discounted value of dividends a maximum. This expected value is given by:

$$
V(S, Z)=E\left(\sum_{t=1}^{\infty} s_{t} v^{t}\right)
$$

The author shows that for $0 \leqslant S \leqslant Z$ we have again an integral equation of Fredholm, namely:

$$
V(S, Z)=v \int_{0}^{Z} V(x, Z) f(x-S) d x+v \int_{Z}^{\infty}(V(Z, Z)+x-Z) f(x-S) d x .
$$

A special case

When we assume as profit distribution:

$$
\begin{cases}f(x)=k \alpha e^{-\alpha x} \quad x>0 \\ f(x)=(\mathrm{I}-k) \alpha e^{\alpha x} \quad & x \leq 0 \\ & \frac{1}{2}<k<\mathrm{I},\end{cases}
$$

rather simple analytical expressions for $D(S, Z)$ and $V(S, Z)$ are obtained. Now the function $V(S, Z)$ can easily be differentiated 
with respect to $Z$ and it appears that there is a unique maximum which is independent of the initial capital $S$. The author remarks, that probably this last result does not hold in general.

Finally he gives in two tables numerical values as regards $D(S, Z)$ and $V(S, Z)$.

It is evident that both wishes with regard to $D(S, Z)$ and $V(S, Z)$ cannot be fulfilled at the same time as they "counteract each other". However, the real problem consists of a sensible, balanced combination of the demands. Theoretically speaking, we have again the question of defining a utility function:

$$
U(S, Z)=U_{D}(S, Z)+U_{V}(S, Z),
$$

and calculating, for a given initial capital $S$, the value of $Z$ for which $U(S, Z)$ is a maximum.

Remark: Wolff treats-with slight modifications-this problem in his new book, I mentioned already (see: chapter V, especially pp. 237-239). Instead of considering the utility of the expected life of the company, he pays attention to the utility of the ruin of the company, what is almost the same as he uses implicitly the discount function. Besides he considers the insurance process to be continuous.

In practice it may be better to look at such tables as Borch gives. If we have extensive tables, e.g. for several "acceptable" profit distributions and reinsurance contracts, we may perhaps get a slight idea how things are. This alone is worthwhile, as it may influence future managements in a right way.

However as the author says, the main problem is to spell out what the company wants in an unambiguous way. "The general manager and his board must solve this problem, without much help from actuaries and other experts on operations research".

\section{G. de Leve and P. J. Weeda. Driving with Markov-programming}

This morning a lecture has been given to us by Mr. Weeda on "driving with Markov-programming". Therefore it seems rather superfluous to report-in the strict sense of the word-on the contribution of de Leve and Weeda. Nevertheless allow me to make some remarks in relation with another study, made in the past on bonus systems. 
Several important papers on automobile insurance have-in the course of the years - been published in ASTIN-bulletin. Especially the colloquium at la Baule in 1959 was devoted to the subject "No claim discount in insurance, with particular reference to motor business". And in ASTIN-bulletin, April I960, we find important publications on bonus systems and related subjects by Franckx, Martin, Philipson and Thyrion. I will draw your attention once more to a paper on bonus systems published in I957 by Ulf Grenander in the Scandinavian yournal, titled "Some remarks on Bonus systems in automobile insurance". Where it treats, in the third part, essentially the same problem as that of de Leve and Weeda, it is proper to compare both studies and point out the differences.

In my opinion, the study of de Leve and Weeda should in the first place be considered as an application in the insurance field of Markov-programming, with the accent strongly on Markovprogramming. As important parts of the common actuarial theory, including life insurance theory, can be reframed in terms of Markov theory, it is clear that actuaries ought to be greatly interested in certain aspects of Markov-programming. Obviously Markovprogramming, although a difficult subject, is a very natural way for attacking certain-frequently occuring-types of decision problems. Hence the study of de Leve and Weeda is certainly a very valuable contribution to ASTIN. Besides the formulation of the decision problem of the motorist in precise terms and the working out of the mathematics involved is impressive. But we actuaries and especially those who are engaged with motor-insurance should not be deceived as de Leve and Weeda only give advice to the motorist and have no pity at all with the insurance companies. The following up of the advice of de Leve and Weeda by the public must necessarily result in a rather bad financial outcome for the insurer. At any rate the insurance company should be watchful as regards possible "strategies" of the public. It is not sure whether the total premium income based on the planned bonus system remains sufficient to cover the claim costs. In the terminology of Markov-programming, the bonus system may become distorted in a high degree as a consequence of replacing the "natural process" by the "decision process". 
Grenander pays attention to this question too. He begins his paper with remarks on setting up bonus systems without considering "game aspects" on the side of the motorist. First of all he considers the equilibrium conditions. The mean premium income should be no less than the expected claim costs. Also the variance of the premium income should be not too large. This again means a restriction for the bonus systems which can be considered. Then he realises that the motorist may take an operations research expert's advice, to minimize his costs of premiums and damages; this is once more a restriction for introducing adequate bonus systems.

I will now remind you briefly of how Grenander tries to solve the problems.

We assume that if an accident happens in class $C_{i}$, a claim is made only if the damage exceeds a value $s_{i}$. In terms of "game theory" the function $s_{i}$ will lead to the optimal strategy for the motorist if the costs on the long run are minimized. By costs on the long run we may understand the expected costs of the random walk or the discounted expected costs as Grenander does. The original transition probabilities $\pi_{i j}$ are by the strategy transformed into the probabilities:

$$
\pi_{i j}(s)=\pi_{i j} \int_{i_{i}}^{\infty} f(x) d x, i, j=\mathrm{I}, \ldots, k,
$$

$f(x)$ the distribution function of the damage. The expected discounted value of future premium and damage costs for a policy in class $C_{t}$ will be (accidents only occur at the end of the year):

$$
\begin{gathered}
F_{i}=p_{i}+v \sum_{j=1}^{k} \pi_{i j}(s) p_{j}+v^{2} \sum_{j=1}^{k} \sum_{h=1}^{k} \pi_{i} h(s) \pi_{h j}(s) p_{j}+\ldots+ \\
\pi \int_{0}^{s_{i}} x f(x) d x+v \pi \sum_{j=1}^{k} \pi_{i j}(s) \int_{0}^{j} x f(x) d x+\ldots
\end{gathered}
$$

with $p_{i} i=\mathrm{I}, \ldots, k$, the premium in class $C_{i}$ and $\pi$ the accident probability.

If we introduce the vectors:

$$
\begin{aligned}
& F(s)=\left(F_{1}(s) . . . . . F_{k}(s)\right) \\
& p=\left(p_{1} \quad \ldots \ldots . p_{k}\right) \\
& \lambda=\left(\begin{array}{lll}
\lambda_{1} & \cdots & .
\end{array} \lambda_{k}\right) \text { with } \lambda_{i}=\int_{0}^{i} x f(x) d x \text {, }
\end{aligned}
$$


we have the matrix-formula:

$$
\begin{array}{r}
F(s)=p+v \Pi(s) p+v^{2} \Pi^{2}(s) p \\
\pi \lambda(s)+v \pi \Pi(s) \lambda(s)+\ldots+
\end{array}
$$

As $v<\mathrm{I}$ this series converges and we obtain:

$$
F(s)=(I-v \Pi(s))^{-1}(p+\pi \lambda(s)) .
$$

If there is a minimum we find it by differentiating $F(s)$ to $s$. It appears that we have to solve the matrix equation:

$$
v \Pi \text {. }(I-v \Pi(s))^{-1}(p+\pi \lambda(s))=s .
$$

In this formula the premium vector $p$ is not a function of the vector $s$. Suppose, that $\bar{s}$ is the optimal strategy. For the expected discounted premium income and the expected discounted claim costs of the insurance company we obtain similar matrix expressions.

For instance the premium income is given by the scalar

$$
I(s)=\sum_{i=1}^{k} n_{i} p_{i}+v \sum_{i=1}^{k} n_{i} \sum_{j=1}^{k} \pi_{i j}(s) p_{j}+\ldots
$$

$n_{i}=$ the number of policies in class $C_{i}$.

Or in matrix notation:

$$
\begin{gathered}
I(s)=n p+n v \Pi(s) p+\ldots \\
n v^{2} \Pi(s) p+\ldots \ldots=n(I-v \Pi(s))^{-1} p .
\end{gathered}
$$

For every bonus system $p$ and corresponding optimal strategy $\bar{s}$ we may now compare $I(\bar{s})$ with $U(\bar{s})$. We should be careful that for a certain range of the risk parameter (accident-frequency) $\pi$, the inequality:

$$
I(\bar{s})>U(\bar{s}) \text { holds }
$$

In fact this is what Grenander does.

Remark: Grenander actually considers financial equilibrium conditions for the stationary situation and does not apply the equivalence principle for discounted values. This difference however does not influence our considerations in an essential way. 
Now we may return to the study of de Leve and Weeda and may ask:

I. whether the methods used by them, disregarding the discount factor, lead for given bonus systems approximately to the same optimal strategy $\bar{s}$,

2. what are the values of $I(s)-U(s)$ for certain types of decision processes and especially for the optimal strategy $\bar{s}$ for some existing bonus systems and-theoretical-claim distributions.

By answering these two questions I think interesting information becomes available for the insurance companies.

d'Hooge, Franckx and Gennart. Utilisation pratique de la méthode de simulation dans l'assurance "non-life"

The study of d'Hooge, Franckx and Gennart is largely a technical report with many figures, tables and graphs on the outcome of a simulation technique for the distribution of claim costs. A main reason that the authors take resort to Monte Carlo methods is the wish to avoid the wellknown difficulties of computing convolutions.

As there is at this time a great need of practical evaluation in the insurance field of operations research methods, with among them Monte Carlo techniques, this report is very welcome. As for the probability model the authors use a claim costs distribution already studied by Delaporte in relation with motor insurance. This compound distribution consists of an empirical distribution for the number $n$ of claims for one policy in a year, and the distribution for the size $m$ of the individual claim, given by

$$
F(m)=P[m<m]=\mathrm{I}-e^{-m}
$$

The costs for the different contracts are considered as identical, independently distributed, random variables. The simulation has been performed for 360.000 policies, and the technical aspects of the technique are described in an instructive way. The simulation for one policy has been carried out by first simulating the number of claims in a year and next, in case this number is $n$, by simulating $n$ times the size $m$ of the claim. Then the sum

$$
s=\sum_{i=1}^{n} m_{i}
$$


is the simulated value of the total costs of the policy. The method for generating values of random variables is thereby the classical one based on the method of producing random numbers in the unit interval.

The authors pay attention to the characteristic properties of the distribution of the total claim costs by calculating the lower moments, the skewness, kurtosis etc. They give extensive tables of them for increasing size $N$ of the samples and the number of contracts $k$. Also they compare, by graphical means, (probability paper), the outcome of the simulation with the normal distribution and some other well-known empirical distributions for a fixed number of contracts $(k=\mathrm{IOO})$.

After these questions of, let us say, identifying the simulated distribution, the authors turn to such important practical questions as calculating the probabilities of excess and premiums for "stop loss" reinsurance. It is not quite possible to give here full information as regards the rich contents of this paper. However, it is important to mention the conclusions to which the authors come, namely:

a) The simulation technique is very laborious and expensive. If we only need information as regards the lower moments of the distribution and functions of them such as the coefficient of variation, direct calculations should be preferred.

b) The method of simulation is very valuable in judging the accuracy of the approximation by the normal distribution.

c) For rather small numbers of policies simulation still gives the possibility of prediction and interpolation.

\section{Final remarks}

The papers submitted clearly show that the operations research methods have only value if the ends of the company and the insured people can be defined in a realistic manner. Borch stresses especially this point and remarks that for the solution of the important general decision problems, not lying in the technical sphere, which the company faces, the operations research expert is perhaps of little help. Besides, although the mathematics involved with operations research is very interesting, it can also be rather mis- 
leading. To this I would add that in particular mingling of orthodox actuarial concepts and techniques of operations research is dangerous, as it may obscure very important liquidity aspects.

Actuaries brought up in the private life insurance business stick to the equivalence principle, i.e. comparing discounted values of premiums, costs and benefits. Introducing the discount factor into the calculations actually means - in the terminology of Borch-the introduction of a special preference ordering for the payments of subsequent years. We may ask ourselves whether the "utility function" $v^{t}$ describes the different preferences in a right way. Merely stating that the function $v^{t}$ may be replaced by another function is of course not wholly satisfying. Moreover what is the influence of the value of the discount factor, on the outcome of the optimalisation problem? In addition there may be some liquidity demands. To turn back to the examples we dealt with; there may be problems of shortage of cash on the side of the motorist and of investments for the company. Although the techniques of operations research are promising, there remains the important question of evaluating them for the practical use in the insurance field. Before definitely using a certain technique and mathematical model, it seems wise to perform calculations for alternative, detailed, models and to compare the results.

This last remark also applies to Monte Carlo methods as shown in a realistic manner by the simulation technique for claim costs distributions reported on. We may conclude that we actuaries have come in the stage of experimenting with operations research techniques.

\section{Received afterwards:}

Jan Ohlin, A generalization of a result by Borch and Kahn on the optimal properties of stop loss reinsurance.

The study of Ohlin implies an extension of the results of Borch and Kahn on the optimal property of stop loss reinsurance in that this reinsurance scheme minimizes the variance of the remaining insured amount. 
OPERATIONS RESEARCH AND MODERN ECONOMIC THEORY 6I

Ohlin proves two things:

a) The property of stop loss reinsurance of minimizing the variance holds true for a quite general class of measures of dispersion.

b) With regard to this property, nothing is gained by allowing the reinsurance scheme to depend on the claims of the individual policies. 